American Journal of Applied Sciences 4 (2): 94-98, 2007

ISSN 1546-9239

(C) 2007 Science Publications

\title{
Numerical Analysis of Forward Expansion Characteristics of Ablated Plasma by Pulsed Ion-Beam Interaction with Al Target
}

\author{
Chainarong Buttapeng, Masaru Yazawa and Nobuhiro Harada \\ Department of Electrical Engineering, Nagaoka University of Technology \\ 1603-1 Kamitomioka, Nagaoka, 940-2188, Japan
}

\begin{abstract}
The main objective of the current study was to investigate the behavior of ablation plasma which was produced by irradiating pulsed ion-beam with a solid target of $\mathrm{Al}$ thin foil. The technique used here was called a forward expansion---beams irradiation and the ablation plasma expansion are in an opposite direction. A one-dimensional hydrodynamic model was used to simulate the dynamic of the target with a thickness of $9 \mu \mathrm{m}$ and the plasma. In this model, the internal energy together with both of the latent heat of fusion phenomenon and the latent heat of vapor are also taken into account. Physical parameters are presented in terms of ablated plasma temperature, pressure and the energy deposition distribution.
\end{abstract}

Key words: Ablated plasma, numerical analysis, pulsed ion-beam

\section{INTRODUCTION}

Ion-beams have been used for various applications, particularly in material sciences, such as evaporation, ion implementation and ion sputtering depending on voltage and current. For example, several megavolts intense pulsed ion-beam produces a high-temperature and high- density ablation plasma through fusion, vaporization and ionization by the ion energy deposition that is penetrated into the solid material ${ }^{[1-3]}$. The ion-beams are able to form ablation plasmas efficiently, because absorption and beam generation is better than that of laser. Using these characteristics, we have reported tests of a possible application by flyer acceleration using the reaction and expansion of ion beam ablation plasma ${ }^{[4,5]}$. At this university, the research has been conducting of both numerical simulation and experiment. Yatsui et al reported that a flyer velocity of $7.7 \mathrm{~km} / \mathrm{s}$ was achieved by irradiating an intense pulsed ion-beam energy density of $2 \mathrm{~kJ} / \mathrm{cm}^{2}$ with $\mathrm{Al}$ foil thickness of $50 \mu \mathrm{m}^{[1,2]}$. We then developed a numerical tool for the analysis of flyer acceleration which is produced by pulsed ion beam irradiating with Al thin foil ${ }^{[4]}$. In this study, the new technique of forward expansion is introduced, while the numerical procedures are still based on the ion beam-solid material interaction concept. The advantage of this new concept is that it is able to understand the ablated plasma production phenomenon clearer, because the ion-beam irradiation and the plasma expansion are in the opposite direction. The set of one-dimensional hydrodynamic equations together with the equation of state of an ideal gas are used. Internal energy and the latent heat of a fusion and the latent heat of a vapor of a target material are also taken into account.

Principle and modeling: The basic concept of the forward expansion of ablation plasma by the interaction of pulsed ion-beam and the solid target is shown in Fig. 1. When we irradiate Al target with pulsed ionbeam, which is produced by the pulse power generator, ablation plasma is formed as soon as the ion beam energy penetrates the target to the certain depth. Because the ablation plasma produces extremely high pressure at a position closest to the final depth of $9 \mu \mathrm{m}$, therefore, ablation plasma with high pressure or high momentum expands to the opposite direction of the ion beam irradiation. This ablation plasma acts as the exhaust momentum to drive space probes forward ${ }^{[5]}$.

We use a one-dimensional fluid model in order to simulate the dynamics of the target and the ablation plasma while incident ion beams interact with the target ${ }^{[3,4]}$. Different parts of the target are in solid, liquid and gaseous and also plasma form, which is treated as a compressible fluid without any charge effects. This approximation is acceptable because the ratio of the Debye length to the electron mean free path is much less than 1 for an electron density of about $10^{23} \mathrm{~cm}^{-3}$ and the electron temperature is several tens of $\mathrm{eV}$ $\left(10^{6} \mathrm{~K}\right)$.

Moreover, the ionization process can be expressed by Saha's equation because the ablation plasma is considered to be a local thermal equilibrium under the extremely high pressure ${ }^{[5,6]}$. Therefore, the basic hydrodynamic equations ${ }^{[3]}$ employed are:

$$
\frac{\mathrm{D} \rho}{\mathrm{Dt}}+\rho \frac{\partial \mathrm{u}}{\partial \mathrm{x}}=0
$$

Corresponding Author:

Chainarong Buttapeng, MHD \& Plasmadynamics Lab., Department of Electrical Engineering, Nagaoka University of Technology, 1603-1 Kamitomioka, Nagaoka, 940-2188, Japan, Tel: +8125847 9511, Fax: +81258479500 
$\rho \frac{\mathrm{Du}}{\mathrm{Dt}}=-\frac{\partial(\mathrm{P}+\mathrm{q})}{\partial \mathrm{x}}$

$\frac{\mathrm{DU}}{\mathrm{Dt}}=-(\mathrm{P}+\mathrm{q}+\mathrm{U}) \frac{\partial \mathrm{u}}{\partial \mathrm{x}}+\mathrm{S}+\mathrm{H}$

$\mathrm{U}=\mathrm{C}_{\mathrm{v}} \operatorname{Tn}_{\mathrm{i}}{ }^{0}+\sum_{\mathrm{z}=1}^{\mathrm{n}} \mathrm{n}_{\mathrm{i}}^{\mathrm{z}} \varepsilon_{\mathrm{z}}+\left(\mathrm{L}_{\mathrm{f}(\mathrm{T})}+\mathrm{L}_{\mathrm{v}(\mathrm{T})}\right) \mathrm{n}_{\mathrm{i}}{ }^{0}$

Where $\rho$ is the target mass density and $u$ is the relative velocity. $\mathrm{P}$ is pressure, $\mathrm{T}$ is temperature and $q$ is Von Neuman viscosity. $C_{v}$ represents the specific heat at constant volume. $\mathrm{S}$ indicates energy deposition (stopping power) of the ion beam based on the ion beam-target interaction ${ }^{[4,5]}$. In addition, the term $\mathrm{H}$ denoting energy loss due to thermal conductions is also taken account. $U$ is the internal energy, while $L_{f}$ and $L_{v}$ are the latent heat of a fusion and the latent heat of a vapor. The $\mathrm{n}_{\mathrm{i}}$ defines an ion number density and $\varepsilon$ is for ionization energy. The subscripts $a b$ and $z$ are the ablation plasma and the ionization number, respectively

According to the fact that the ablation plasma is produced instantaneously, the phase changed effects and electromagnetic effects are ignored ${ }^{[7,8]}$. All the above equations are solved by a difference method in the Lagrange scheme ${ }^{[3,4]}$. The physical parameters used in the simulation are listed in Table 1 and are based on the experimental conditions of a pulse power generator "ETIGO II" installed at the Extreme Energy-Density Research Institute, Nagaoka University of Technology, $\operatorname{Japan}^{[9,10]}$.

Table 1: Simulation parameters

Ion Beam Energy Density $\left[\mathrm{J} / \mathrm{cm}^{2}\right]$

Ion Beam Kinetic Energy [MeV]

Pulse Duration [ns]

Beam Particles

Target Material

Target Thickness

100
0.7
60
protons
Aluminum
$9 \mu \mathrm{m}$

\section{RESULTS AND DISCUSSIONS}

Once a pulsed ion beam with energy density of 100 $\mathrm{J} / \mathrm{cm}^{2}$ at the pulse duration of $60 \mathrm{~ns}$ reaches Al target, the part of the target is melted, evaporated and is instantaneously ionized forming high-density, highpressure and high-temperature ablation plasma. The 0.7 $\mathrm{MeV}$ of accelerating voltage was able to penetrate ionbeam energy to almost the certain depth of the Al foil of $9 \mu \mathrm{m}$. The ablated plasma afterward heated and expanded to the opposite direction of the irradiation. Figure 2 shows the ion density of ablation plasma after expanding to the opposite side. The point where $\mathrm{x}=0$ and $\mathrm{y}=0$ is the edge of $\mathrm{Al}$ foil. With an initial irradiating of $10 \mathrm{~ns}$, ion-beam deposits its energy strongly and high density of ion is found at this stage. The ion-beam energy somehow decreases after $15 \mathrm{~ns}$; because most of the energy is afterward spent for the high-temperature formation process. Ablated plasma continues its formation process, while increasing the rate of ion- beam energy penetration through the expanding phenomenon as shown in Fig. 3. Ion-beam energy terminates its penetration at $60 \mathrm{~ns}$, which is as equal as

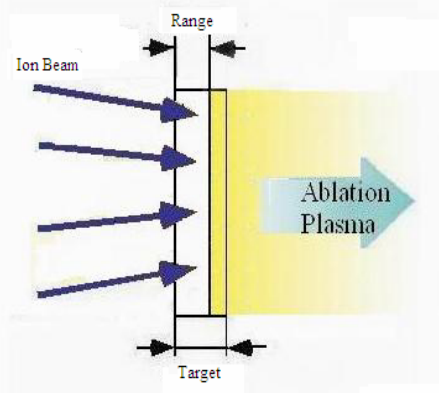

Fig. 1: Ion-beam and solid interaction concept for the forward ablation plasma expansion

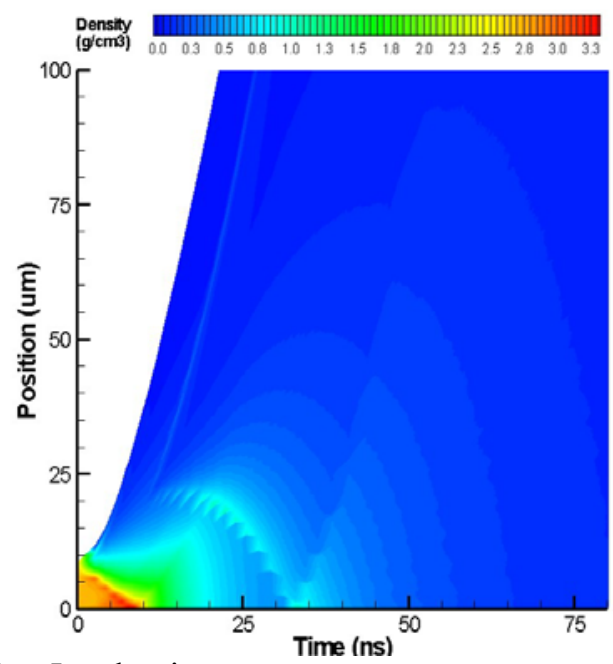

Fig. 2: Ion density

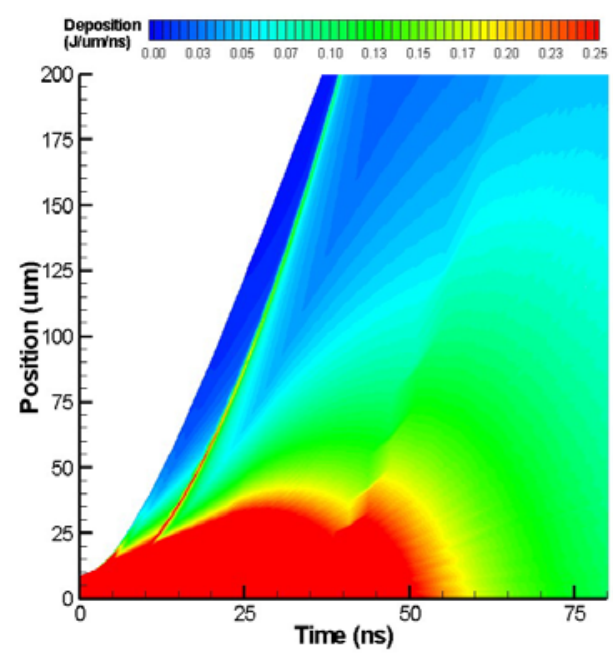

Fig. 3: Ion-beam deposition

its pulse duration. High-energy density ablation plasma is found at approximately $25 \mu \mathrm{m}$ far from the edge of the target. This high-energy ablation keeps continue and transforms into the ionization process at approximately $15 \mathrm{~ns}$ to form high-temperature plasma as shown in Fig. 
4 and 5. The more the ionization process appears, the higher ablated plasma temperature is observed and is in the range of 3 to $3.5 \mathrm{eV}$.

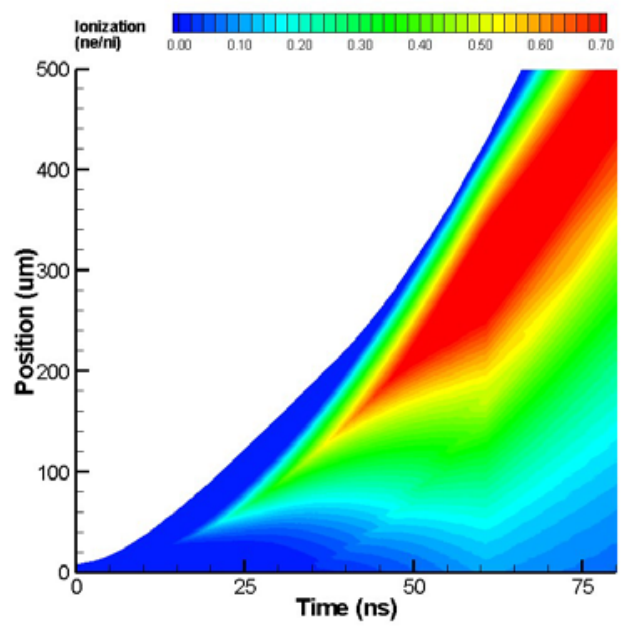

Fig. 4: Ionization

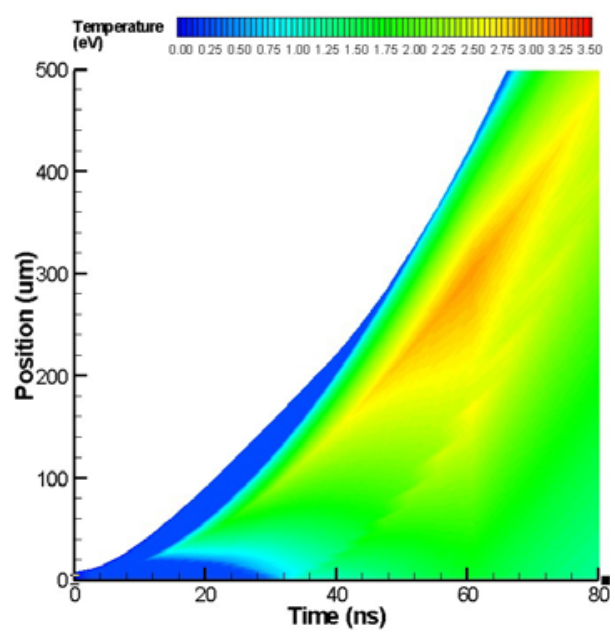

Fig. 5: Ablation plasma temperature

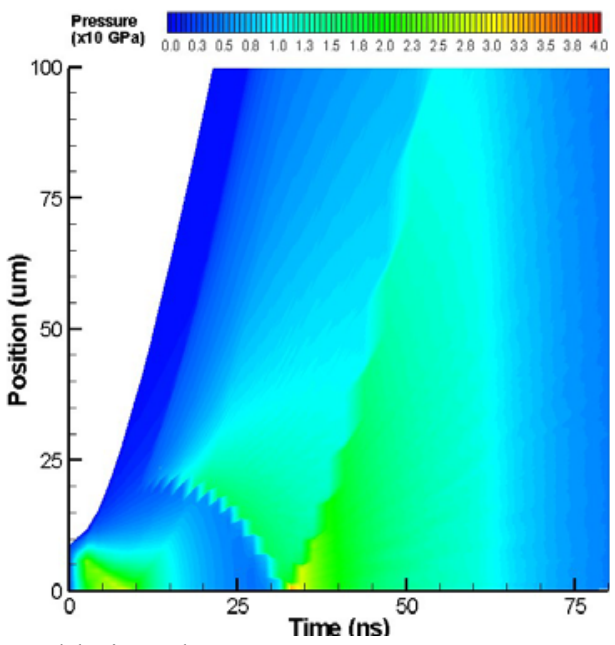

Fig. 6: Ablation plasma pressure
Figure 6 shows the ablation plasma pressure distribution after expanding to the opposite direction. One can see that the pressure trends to sharply increase and gradually fall instantaneously. At the period of

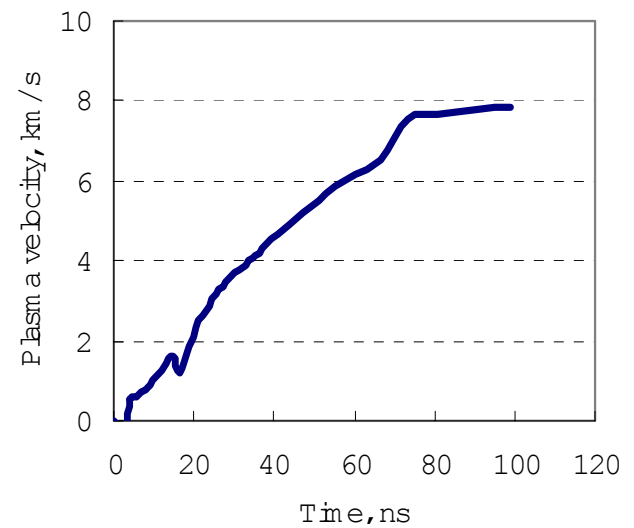

Fig. 7: Plasma velocity

about $20 \mathrm{~ns}$ as soon as the ablated plasma is formed, ablated plasma increases its pressure rapidly to form high-pressure ablation plasma. The pressure at this stage is in the range of 10-27 GPa. During the period of 20-30 ns, the pressure of the ablated plasma decreases as some of ion-beam energy is spent into the vaporization process. The main factor causes the decreased of ablated plasma pressure is due to the constant ion-beam current for plasma formation and expansion at the beginning of the ion-beam irradiation. The pressure of ablated plasma starts increasing once again at approximately $30 \mathrm{~ns}$ because of the propagation of the strong shock-wave that returns to the edge of a target. The high-pressure ablated plasma plumed consumes the area of approximately $6250 \mu \mathrm{m}^{2}$.

Maximum pressure of approximately $35 \mathrm{GPa}$ at 30 ns is observed. Not only has the ablation plasma pressure increased with time through its expansion, but also the ablated plasma velocity. The ablated plasma velocity trend is shown in Fig. 7. Plasma reaches the peak velocity of approximately $7.9 \mathrm{~km} / \mathrm{s}$ at the ionbeam irradiation of $75 \mathrm{~ns}$. This value of peak velocity seems to be constant afterward. We can say that this obtained velocity is the a maximum plasma velocity of $\mathrm{Al}$ foil with the thickness of $9 \mu \mathrm{m}$. The plasma velocity trend shown in Fig. 7 is also considered to be more viable and much better than that of obtained in the previous study at the same energy input of 100 $\mathrm{J} / \mathrm{cm}^{2[4,5,8]}$. It is too obvious that the physical characteristics of ablated plasma by the forward expansion concept show clearer understanding of the production mechanism, its expansion phenomenon and so is the plasma velocity.

\section{CONCLUSION}

This study investigated the simulation model of forward expanding ablation plasma which is produced by irradiating pulsed ion-beam with an $\mathrm{Al}$ foil. The set 
of one-dimensional hydrodynamic equations are used. The latent heat of both fusion and vaporization process, which are not included in the previous works, were also taken into account. This consideration was able to understand the ablated plasma formation and expansion clearer. Because the plasma formation process and the ion-beam irradiation were in opposite direction, the characteristics of ablation plasma understood clearer than those of obtained by the flyer acceleration concept.

\section{REFERENCES}

1. Yatsui, K., H. Shinkai, K. Kashine, W. Jiang, M. Kagihiro and N. Harada, 2001. Foil acceleration by intense pulsed ion beam ablation plasma. Jpn. J. Appl. Phys., 40: 955-959.

2. Kashine, K., M. Yazawa, N. Harada, W. Jiang and K. Yatsui, 2002. Foil acceleration of double-layer target by intense pulsed ion beam ablation. Jpn. J. Appl. Phys., 41: 4014-4018.

3. Duderstadt, J.J. and G.A. Moses, 1982. Inertial Confinement Fusion. Wiley. New York, pp: 65-179.

4. Yazawa, M., C. Buttapeng, N. Harada, H. Suematsu, W. Jiang and K.Yatsui, 2006. Formation and expansion of ablation plasma produced by pulsed ion beams for thin films production. The journal of the Institute of Electrical Engineers of Japan: Transaction on Fundamentals and Materials, 126: 45-50.
5. Yazawa, M., C. Buttapeng, N. Harada, H. Suematsu, W. Jiang and K.Yatsui, 2005. Application to space propulsion with ablation plasma produced by pulsed ion beam. Trans. JSASS Space Tech. Japan., 3: 1-6.

6. Thomas, A., 1981. A finite temperature model for ion energy deposition in ion-driven inertial confinement fusion target. J. Appl. Phys., 52: 6522-6532.

7. Spitzer, L., 1962. Physics of Fully Ionized Gases. Wiley. New York.

8. Harada, N., M. Yazawa, K. Kashine, W. Jiang and K. Yatsui, 2001. Development of a numerical tool for foil acceleration by an intense pulsed ion beam. Jpn. J. Appl. Phys., 40: 960-964.

9. Yatsui, K., A. Tokuchi, H. Tanaka, H. Ishizuka, A. Kawai, E. Sai, K. Masugata, M. Ito and M. Matsui, 1985. Geometric focusing of intense pulsed ion beam from racetrack type magnetically insulated diodes. Laser and Particle Beams, 3: 119-155.

10. Jiang, W., T. Sakagami, K. Masugata and K.Yatsui, 1995. Three dimensional tight focusing of intense pulsed light-ion beam by spherical plasma focus diode. Laser and Particle Beams, 13: 343-350. 
Am. J. Applied Sci., 4 (2): 94-98, 2007

\section{All articles Published with Science Publications are peer - reviewed and available at

$$
\text { www.scipub.org }
$$ \\ Submit your next article to us}

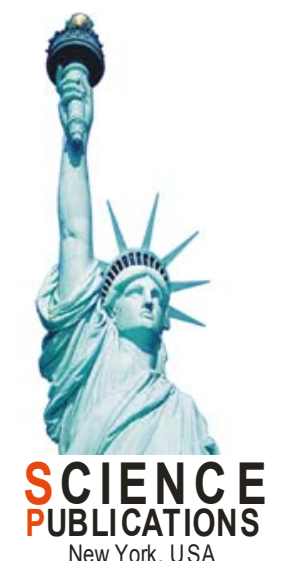

